\title{
Surface-associated proteins of Staphylococcus aureus: Their possible roles in virulence
}

\author{
T.J. Foster * and D. McDevitt \\ Microbiology Department, Moyne Institute, Trinity College, Dublin 2, Ireland
}

(Received 10 January 1994; revision received 21 February 1994; accepted 22 February 1994)

\begin{abstract}
A class of proteins that are associated with the cell surface of Gram-positive bacteria has been recognised. Common structural features which are implicated in the proper secretion and attachment of these proteins to the cell surface occur in the C-termini. N-terminal domains interact with the host by binding to soluble host proteins, to matrix proteins or to host cells. They probably have important roles in pathogenicity by allowing bacteria to avoid host defences and by acting as adhesins. Four such proteins of Staphylococcus aureus have been characterised: protein A (immunoglobulin binding protein), fibronectin binding proteins, collagen binding protein and the fibrinogen binding protein (clumping factor). Site-specific mutants are being used to define their roles in pathogenesis in in vitro and in vivo models of adherence and infection.
\end{abstract}

Key words: Staphylococcus aureus; Clumping factor; Protein A; Fibronectin binding protein; Collagen binding protein; Fibrinogen binding protein

\section{Introduction}

It is now recognised that pathogenic Grampositive bacteria express, on their surfaces, proteins which contribute to virulence. They do so by promoting adherence to host cells and/or tissue components and by binding soluble components of plasma such as albumin and immunoglobulins to allow evasion of immune responses and avoidance of phagocytosis. Some of these proteins can act as adhesins performing functions associated

\footnotetext{
* Corresponding author.
}

with fimbriae and outer membrane protein adhesins in Gram-negative bacteria. This article will review the general features of wall-associated proteins before discussing the properties of the four proteins from Staphylococcus aureus that have been implicated in virulence and characterised at the molecular level.

Genes for the immunoglobulin binding protein, protein $\mathrm{A}$, the fibronectin binding proteins, the collagen binding protein and the fibrinogen binding protein have all been sequenced. The ligand binding domains of the proteins have been identified and in some cases analysed in great detail. Their possible roles in the pathogenesis of staphylococcal infections have been studied using 
site-specific mutations in both in vivo and in vitro models of infection.

\section{Features of wall-associated proteins of $S$. aureus}

The sequences of genes for more than fifty wall-associated proteins from different Grampositive bacteria are known and it is apparent that the majority of proteins exhibit a number of common features [1-3]. These proteins are characterised by a signal sequence at the $\mathrm{N}$-terminus (which is cleaved during secretion across the plasma membrane) and at the C-terminus the motif LPXTG preceding a hydrophobic membrane-spanning region and a positively charged tail which is thought to act as a stop-secretion signal (Fig. 1). The LPXTG sequence is important for accurate sorting of the proteins and in some cases is probably cleaved proteolytically to link the $\mathrm{N}$ terminal region covalently to peptido- glycan $[1,2]$. There is evidence to suggest that some wall-associated proteins are not linked to peptidoglycan but remain attached to the plasma membrane [2]. Alternatively, in streptococci some proteins are neither covalently linked to peptidoglycan nor anchored to the membrane [3] and are readily released from protoplasts without detergent treatment. N-terminal to LPXTG, most wall-associated proteins have sequences that are rich in proline and glycine residues. These are predicted to take up an extended conformation which permits the protein to span the thick cell wall. However, this region varies considerably in length from one protein to another and the minimum requirements for wall association are not known.

Schneewind et al. [2] described analytical techniques to determine if a protein is wall-associated in $S$. aureus, and if it is covalently bound to peptidoglycan or is anchored to the membrane. Bacteria growing in defined medium are pulse-

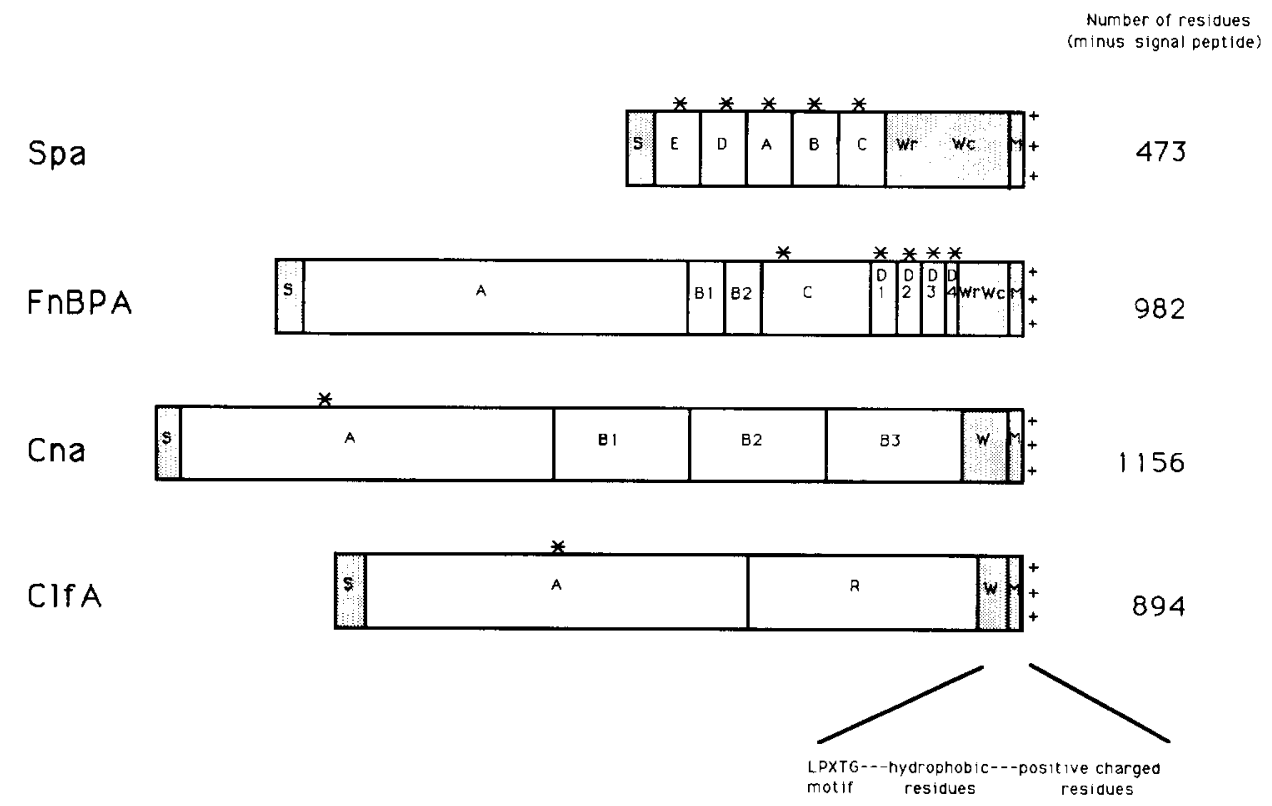

Fig. 1. Organisation of surface associated proteins. The domain organisation of protein A (Spa), the fibronectin binding protein (FnBPA) the collagen binding protein (Cna) and the fibrinogen binding protein (clumping factor, ClfA). The signal sequences (S) are removed during secretion across the cytoplasmic membrane. Each protein has common features at the C-terminus: LPXTG motif, membrane anchor $(\mathrm{M})$ and positively charged residues. Region $\mathrm{W}$ is the putative peptidoglycan spanning region. ${ }^{*}$ indicates the position of the ligand binding domain(s). 
labelled with ${ }^{35} \mathrm{~S}$-methionine for a short period followed by a longer chase. The cells are fractionated by enzymatic digestion with a muramidase (cleaving the peptidoglycan backbone) or an endopeptidase (cleaving the pentaglycine bridge of peptidoglycan). If the protein is linked to the membrane it will be released into hot SDS by either enzyme. If it is covalently linked to peptidoglycan the muramidase will release the protein with peptidoglycan fragments attached to the Cterminus. This causes the protein to migrate as a series of higher molecular mass bands. Further treatment with lysostaphin allows the protein to migrate normally.

\section{Protein A}

Protein A is the archetypal cell wall-associated protein of $S$. aureus and indeed of Gram-positive bacteria. It comprises five (in some isolates four) repeated units of 58 amino acid residues each of which can bind to the Fc region of IgG [4] (Fig. 1). X-ray crystallographic analysis of repeat unit $\mathrm{B}$ complexed with the $\mathrm{Fc}$ fragment demonstrated that two $\alpha$-helices make several contacts with the $\mathrm{C}_{\mathrm{H}} 2$ and $\mathrm{C}_{\mathrm{H}} 3$ domains of the $\mathrm{Fc}$ fragment [5]. Comparison of the amino acid sequences of each repeat suggests that protein $\mathrm{A}$ evolved by tandem duplication events followed by genetic drift resulting in a gradient of homology seen when the repeats are compared [4]. Furthermore, the $\alpha$ helices are more highly conserved than the rest of the repeats and the residues that make contact with residues in the Fc fragment are almost completely identical. This suggests that strong selective pressure has been applied for retention of the $\alpha$-helices and their Fc binding activity. The argument that binding to IgG is a biologically important phenomenon is supported by the finding that two IgG binding proteins of streptococci (protein $\mathrm{G}$ and protein $\mathrm{H}$ ) with structures that are unrelated to protein $A$, bind to precisely the same region of the $\mathrm{Fc}$ fragment [6]. This is a clear example of convergent evolution.

It was suggested that protein A (Spa) and immunoglobulin binding proteins from other pathogens promote coating of the bacterial sur- face with IgG molecules attached by the Fc region which would not be recognisable by the $\mathrm{Fc}$ receptors on leucocytes. The coating of IgG could also mask binding sites for opsonins present in normal serum. In vitro opsonophagocytosis experiments with human polymorphonuclear leucocytes were performed with a $\mathrm{Spa}^{-}$deletion mutant isolated by allelic replacement and an otherwise isogenic wild-type strain [7]. The uptake of wild-type bacteria in the presence of normal human serum was significantly lower than with the isogenic $\mathrm{Spa}^{-}$mutant, suggesting that protein A does indeed hinder opsonisation.

Infection experiments in mice with the protein A-deficient mutant showed that protein $\mathrm{A}$ is a virulence factor. In both subcutaneous abscess and peritonitis infections the Spa mutant was less virulent than the isogenic parental strain [8]. However, no differences were seen in intramammary infections in mice unless the parental strain expressed very high levels of protein A [9]. A mutant deficient in the global regulator Agr was virulent in the mouse mastitis model. It expressed a 50-fold higher level of protein $\mathrm{A}$ than the $\mathrm{Agr}^{+}$ parent but was defective in $\alpha$-toxin and expressed reduced levels of $\beta$-toxin, the main virulence factors for wild-type $S$. aureus in intra-mammary infections [9]. Derivatives of the $\mathrm{Agr}^{-}$mutant lacking protein $\mathrm{A}$ and/or $\beta$-toxin were less virulent than the parental strain suggesting that virulence was due to a combination of the residual $\beta$-toxin and the enhanced level of protein A.

\section{Fibronectin binding proteins}

S. aureus strains 8325-4 and Newman have two closely linked genes that express cell wall-associated fibronectin binding proteins (FnBPA and FnBPB). Some strains of $S$. aureus carry a single $f n b$ gene. Region $\mathrm{A}$ at the N-terminus of FnBPA and FnBPB share only $45 \%$ identical residues but regions $\mathrm{C}, \mathrm{D} \mathrm{W}$ and $\mathrm{M}$ are $95 \%$ similar [10,11] (Fig. 1). The fibronectin (Fn) binding region has been located in repeats D1-D3. A recombinant D1-D3 protein expressed in E. coli could bind Fn in ligand binding assays and could block the attachment of soluble Fn to bacteria. Similarly, 
synthetic peptides corresponding to individual repeats also blocked binding. Another Fn binding activity was detected in region $\mathrm{C}$, possibly at the EEDT motif that has been implicated in the reaction with Fn [11].

The minimum Fn binding region of the 38 residue $D$ repeat units was shown to occur within residues $15-36$ by chemical modification experiments (which identified glutamate and aspartate as important) and with synthetic peptide inhibition experiments [12].

By comparing the amino acid sequences of ligand binding domains of staphylococcal and streptococcal FnBPs, a core fibronectin binding sequence was identified [13]: EDT/S - $(X 9,10)$ GG - $(\mathrm{X} 3,4) \mathrm{I} / \mathrm{VDF}$. The role of this motif in fibronectin binding was tested by adherence blocking experiments using a series of synthetic peptides with variant residues at core positions. Contrary to the original hypothesis, the EDT motif seen in the staphylococcal FnBPs was shown not to be required for binding activity whereas the conserved GG dipeptide and the I/VDF sequence together with additional acidic residues were required [13]. The importance of these residues in promoting adherence of $S$. aureus cells to fibronectin could be confirmed by isolating site-directed mutants expressing FnBPs with specific changes to these motifs.

The ability of bacteria to adhere to biomaterial surfaces that have become coated with host plasma and matrix proteins such as fibrinogen and fibronectin is a major determinant for initiating a foreign body infection. Vaudaux et al. [14] showed that ex vivo human catheters harbour substantial amounts of both blood proteins. However, the fibrinogen was degraded and could not promote bacterial attachment in vitro, whereas the (albeit partially) degraded fibronectin was still able to promote bacterial attachment. It was presumed that the $S$. aureus binding domain in the $27 \mathrm{kDa} \mathrm{N}$-terminal fragment of Fn remained intact.

Fibronectin is a component of blood clots. Clots established in vitro without Fn have a reduced ability to promote staphylococcal attachment [15]. Fn is also found on the surface of epithelial and endothelial cells and may be a factor in promoting bacterial colonisation in CAPD (continuous ambulatory peritoneal dialysis) peritonitis, invasive endocarditis and ruminant mastitis.

We have tested the role of FnBPs in foreign body infection by isolating mutants with specific insertions in both $f n b A$ and $f n b B$ by allelic replacement (C. Greene, D. McDevitt, P. Francois, P. Vaudaux and T.J. Foster, unpublished). The single fnb mutants had reduced ability to bind to Fn-coated plastic surfaces while the double mutant was almost completely defective. This demonstrates that both genes are expressed in $S$. aureus and that both proteins contribute to the ability of $S$. aureus to adhere to Fn-coated biomaterial in vitro. The double mutant was also defective in adherence to coverslips that had been removed from subcutaneously chambers implanted in guinea pigs, an ex vivo foreign body model. This indicates that FnBPs contribute to adherence to biomaterial that has been coated with host proteins in vivo. This could be confirmed by binding experiments with ex vivo human catheters containing partially degraded Fn.

As yet, the only experiments in vivo with Fn binding defective mutants are those reported by Kuypers and Proctor [16] with a derivative of strain $879 \mathrm{RF}$ isolated by transposon (Tn918) mutagenesis. The mutant had a markedly reduced ability to adhere to traumatised rat heart valves, indicating that Fn binding is important in this model of endocarditis. We have shown by Southern blotting and DNA sequencing that the transposon is located $50 \mathrm{bp} 5^{\prime}$ to the single fnb gene in a putative promoter region (C. Greene, D. McDevitt and T.J. Foster, unpublished data). Thus the $f n b$ gene is intact but it is presumably not transcribed.

Antibodies raised against a fusion protein containing regions D1-D3 promoted opsonophagocytosis in vitro and, when administered passively to mice, it promoted clearance of bacteria from the bloodstream [17]. Immunisation of rats with a fusion protein containing region D1-D3 of FnBP protected against experimental endocarditis [18] and, furthermore, when used as a vaccine with an immunostimulation complex, the fusion protein offered some protection to cows against mastitis 
[19]. Thus antigens from FnBPs could be used as components of a vaccine against staphylococcal infections.

\section{Fibrinogen binding protein}

$S$. aureus cells form macroscopic clumps when a suspension is mixed with plasma. The clumping reaction is due to the avid binding of the dimeric plasma protein fibrinogen $(\mathrm{Fg})$ to the clumping factor on the bacterial cell surface. Blocking experiments with fragments of $\mathrm{Fg}$ and with synthetic peptides showed that the $S$. aureus binding site is in the $C$ terminus of the $\gamma$ chain of fibrinogen [19].

Binding to solid phase fibrinogen could be important in promoting bacterial attachment to implanted biomaterial (at least in the early stages before degradation occurs) and to damaged tissue and blood clots, while coating the bacterial cell surface could contribute to evasion of host defences.

It has been suggested that a cell-bound form of coagulase is the clumping factor [21]. Coagulase is a predominantly extracellular protein which binds stoichiometrically with prothrombin to activate the proteolytic function characteristic of thrombin. Thus staphylothrombin converts fibrinogen to fibrin resulting in the formation of a plasma clot. Recently it was shown that coagulase could also bind directly to fibrinogen $[21,22]$. In contrast to the reaction with prothrombin which occurs at the N-terminus of coagulase, the interaction with fibrinogen occurs at the C-terminus [22]. In addition, when cell wall extracts were probed with $\mathrm{Fg}$ in ligand affinity blotting, the major activity was shown to be due to a cell-bound form of coagulase. However, a mutant with a deletion in the chromosomal coa gene isolated by allelic replacement retained the clumping phenotype [22]. Conversely, a clumping factor deficient transposon mutant expressed the same extracellular plasma clotting activity as the wild-type (D. McDevitt and T.J. Foster, unpublished). Thus, although coagulase binds to fibrinogen, the fibrinogen binding region cannot be exposed on the bacterial cell surface and cannot be responsible for clumping.
We have cloned and characterised the gene for clumping factor clf $A$ [23]. The mature ClfA protein comprises 896 residues (Fig. 1). Significant amino acid sequence similarity with the fibronectin binding proteins occurs in the $\mathrm{N}$ - and C-terminal sequences associated with secretion and wall-localisation. In addition some similarity occurs in regions A. ClfA lacks the extensive ProGly-rich repeats of the FnBPs but instead has a remarkable dipeptide repeat comprising 154 alternating serine and aspartate residues (Region R, Fig. 1). This sequence could be exclusively associated with the cell wall or it could be exposed on the cell surface, perhaps acting as a 'stalk' to project region A from the cell surface. Expression of recombinant derivatives in $E$. coli showed that the Fg binding domain is located in the non-repeated region A (D.McDevitt and T.J. Foster, unpublished). Also, a synthetic peptide comprising six SD repeats did not inhibit binding of $S$. aureus to $\mathrm{Fg}$ which indicates that the repeat region is not involved in ligand binding.

The clumping factor has been shown to promote attachment of bacteria to Fg coated surfaces in vitro. The Clf ${ }^{-}$mutants are completely defective in adherence and can be complemented by plasmids expressing the ClfA protein. The ClfA mutant was also defective in adherence to a canine arterio-venous shunt that had been exposed to blood for short periods $(5-60 \mathrm{~min})$ ( $P$. Vaudaux, R.A. Proctor, D. McDevitt, T.J. Foster, D. Lew, H. Wabers and S. Cooper, unpublished). In this model, bacterial adherence is exclusively due to adherence to fibrinogen, whereas the ability to bind to fibronectin does not contribute significantly. This suggests that Fg binding is the most important determinant of adherence of $S$. aureus to newly implanted biomaterials. We are currently testing the $\mathrm{ClfA}^{-}$and $\mathrm{FnBP}^{-}$mutants for virulence in endocarditis and other infection models.

\section{Collagen binding protein}

Strains isolated from osteomyelitis and septic arthritis almost always adhere to collagen and to cartilage in vitro. In contrast, many strains iso- 
lated from soft tissue infections do not express this activity. The collagen adherent bacteria produce the surface-located collagen adhesin, Cna. This protein has features at the C-terminus typical of wall-associated proteins [24] (Fig. 1). The Cna protein occurs either as a molecule of 135 or $110 \mathrm{kDa}$ depending on whether there are 2 or 3 copies of the 187 residue $B$ repeat. The collagen binding activity has been localised to a 168-residue portion of region $\mathrm{A}$ by studying the ligand binding activity of recombinant proteins expressed in E. coli [25].

The tissue tropism suggested by the collagen binding activity of clinical isolates was confirmed by in vitro models of adherence [26]. Both antiCna antibodies and recombinant Cna protein blocked adherence of bacteria to cartilage and to collagen substrates. In addition, a null mutant in cna isolated by allelic replacement was less virulent than the parental strain in a mouse model for septic arthritis [27]. This strongly suggests that Cna is a major determinant in the pathogenesis of osteomyelitis and septic arthritis caused by $S$. aureus.

\section{Adherence to other host proteins and to host cells}

$S$. aureus can bind specifically to several other host proteins such as bone sialoprotein, vitronectin, laminin, elastin, heparan, thrombospondin and mucin. Also, adherence to cultured epithelial, mesothelial and endothelial cells and to platelets has been reported. In many cases a bacterial protein has been tentatively implicated in the reaction but none have been characterised at the molecular level. Recently, a surface protein has been isolated which exhibits multifactorial binding to fibrinogen, fibronectin, collagen, thrombospondin and vitronectin via carbohydrate residues on the host glycoproteins [28]. At least in the cases of the first three host proteins this must be a secondary (accessory) adhesin to those described in this review.

\section{Summary and future prospects}

So far only four of the ligand binding phenomena exhibited by strains of $S$. aureus have been analysed at the molecular level. In each case a cell wall-associated protein with ligand binding activity is involved. Protein $\mathrm{A}$ and fibronectin binding protein have multiple binding sites involved in interactions with host proteins (IgG and fibronectin, respectively) while the collagen and fibrinogen binding proteins appear to have single binding domains.

Preliminary experiments with the fibronectin binding protein $D$ repeat region suggest that it might be feasible to use this region to vaccinate ruminants against the economically important disease mastitis [17]. Also, it might be possible to prevent foreign body infection in hospitalised patients with a vaccine comprising FnBP and ClfA. Bone infections might be prevented with a vaccine comprising Cna. An advantage of vaccines directed at surface proteins is that they will block adherence of bacteria as well as promoting phagocytosis of the non-adherent bacteria. $S$. aureus adheres to several different host proteins so it might be necessary to include epitopes from several different binding proteins to be fully effective.

\section{Acknowledgement}

Research in the authors' laboratory is supported by the Wellcome Trust and by the Health Research Board of Ireland.

\section{References}

1 Schneewind, O., Model, P. and Fischetti, V.A. (1992) Sorting of protein A to the staphylococcal cell wall. Cell 70, 267-281.

2 Schneewind, O., Mihaylova-Petkov, D. and Model. P. (1993) Cell wall sorting signals in surface proteins of Gram-positive bacteria. EMBO J. 12, 4803-4811.

3 Kehoe, M.A. (1994) Cell-wall-associated proteins in Gram-positive bacteria. In: New Comprehensive Biochemistry. Bacterial Cell Wall (Ghuysen, J.M. and Hakenbeck, R., Eds.), in press.

4 Uhlen, M., Guss, B., Nilsson, B., Gatenbeck, S., Philipson, L. and Lindberg, M. (1984) Complete sequence of the staphylococcal gene encoding protein A. A gene evolved through multiple duplications. J. Biol. Chem. 259, 16951702. 
5 Deisenhofer, J. (1981) Crystallographic refinement and atomic models of a human $\mathrm{Fc}$ fragment and its complex with fragment B of protein A from Staphylococcus aureus at 2.9- and 2.8 A resolution. Biochemistry 20, 2361-2370.

6 Frick, I.M., Wikstrom, M., Forsen, S., Drakenberg, T., Gomi, H., Sjobring, U. and Bjorck, L. (1992) Convergent evolution among immunoglobulin G-binding bacterial proteins. Proc. Natl. Acad. Sci. USA 89, 8532-8536.

7 Gemmell, C.G., Tree, R., Patel, A., O'Reilly, M. and Foster, T.J. (1990) Susceptibility to opsonophagocytosis of protein A, $\alpha$-haemolysin and $\beta$-toxin deficient mutants of Staphylococcus aureus isolated by allele-replacement. Zbl. Suppl. 21, 231-236.

8 Patel, A.H., Nowlan, P., Weavers, E.D. and Foster, T.J. (1987) Virulence of protein A-deficient and alpha-toxindeficient mutants of Staphylococcus aureus isolated by allele replacement. Infect. Immun., 55, 3103-3110.

9 Foster, T.J., O'Reilly, M., Phonimdaeng, P., Cooney, J., Patel, A.H. and Bramley, A.J. (1990) Genetic studies of virulence factors of Staphylococcus aureus. Properties of coagulase and $\gamma$-toxin and the role of $\alpha$-toxin, $\beta$-toxin and protein A. In: The Pathogenesis of S. aureus Infections in Molecular Biology of the Staphylococci (Novick, R.P., Ed.), pp. 403-417. VCH, Cambridge, New York.

10 Signas, C., Raucci, G., Jonsson, K., Lindgren, P.E., Anantharamaiah, G.M., Hook, M. and Lindberg, M. (1989) Nucleotide sequence of the gene for a fibronectin-binding protein from Staphylococcus aureus: use of this peptide sequence in the synthesis of biologically active peptides. Proc. Natl. Acad. Sci. USA 86, 699-703.

11 Jonsson, K., Signas, C., Muller, H.P. and Lindberg, M (1991) Two different genes encode fibronectin binding proteins in Staphylococcus aureus. The complete nucleotide sequence and characterisation of the second gene. Eur. J. Biochem. 202, 1041-1048.

12 McGavin, M.J., Raucci, G., Gurusiddappa, S. and Hook, M. (1991) Fibronectin binding determinants of the Staphylococcus aureus fibronectin receptor. J. Biol. Chem. 266, 8343-8347.

13 McGavin, M.J., Gurusiddappa, S., Lindgren, P.E., Lindberg, M., Raucci, G. and Hook, M. (1993) Fibronectin receptors from Streptococcus dysgalactiae and Staphylococcus aureus. Involvement of conserved residues in ligand binding. J. Biol. Chem. 268, 23946-23953.

14 Vaudaux, P. Pittet, D., Haeberli, A., Lerch, P.G., Morgenthaler, J.J., Proctor, R.A. Waldvogel, F.A. and Lew, D.P. (1993) Fibronectin is more active than fibrin or fibrinogen in promoting Staphylococcus aureus adherence to inserted intravascular devices. J. Infect. Dis. 167, 633-641.

15 Raja, R.H., Raucci, G. and Hook, M. (1990) Peptide analogs to a fibronectin receptor inhibit attachment of Staphylococcus aureus to fibronectin-coated substrates. Infect. Immun. 58, 2593-2598.
16 Kuypers, J.M. and Proctor, R.A. (1989) Reduced adherence to traumatised rat heart valves by a low-fibronectinbinding mutant of Staphylococcus aureus. Infect. Immun. $57,2306-2312$.

17 Rogalska, B. and Wadstrom, T. (1993) Protective opsonic activity of antibodies against fibronectin-binding proteins (FnBPs) of Staphylococcus aureus. Scand. J. Immunol. 37, $575-580$.

18 Schennings, T., Heimdahl, A., Coster, K. and Flock, J.I. (1993) Immunisation with fibronectin binding protein from Staphylococcus aureus protects against experimental endocarditis in rats. Microb. Pathogen. 15, 227-236.

19 Nelson, L., Flock, J.I., Hook, M., Lindberg, M., Muller, H.P. and Wadstrom, T. (1992) Adhesins in staphylococcal mastitis as vaccine components. Flem. Vet. J. 62 (Suppl 1), $111-125$

20 Hawiger, J., Timmons, S., Strong, D.D., Cottrell, B.A., Riley, M. and Doolittle, R.F. (1982) Identification of a region of human fibrinogen interacting with staphylococcal clumping factor. Biochemistry 21, 1407-1413.

21 Boden M.K. and Flock, J.I. (1989) Fibrinogen-binding protein/clumping factor from Staphylococcus aureus. Infect. Immun. 57, 2358-2363.

22 McDevitt, D., Vaudaux, P. and Foster, T.J. (1992) Genetic evidence that bound coagulase of Staphylococcus aureus is not clumping factor. Infect. Immun. 60, 1514-1523.

23 McDevitt, D., Francois, P, Vaudaux, P. and Foster, T.J. (1994) Molecular characterisation of the fibrinogen receptor (clumping factor) of Staphylococcus aureus. Molec. Microbiol. 11, 237-248.

24 Patti, J.M., Jonsson, H., Guss, B., Switalski, L.M., Wiberg, K., Lindberg, M. and Hook, M. (1992) Molecular characterisation and expression of a gene encoding Staphylococcus aureus collagen adhesin. J. Biol. Chem. 267, 4766-4772.

25 Patti, J.M., Boles, J.O. and Hook, M. (1993) Identification and biochemical characterisation of the ligand binding domain of the collagen adhesin from Staphylococcus aureus. Biochemistry 32, 11428-11435.

26 Switalski, L.M., Patti, J.M., Butcher, W., Gristina, A.G., Speziale, P. and Hook, M. (1993) A collagen receptor on Staphylococcus aureus strains isolated from patients with septic arthritis mediates adhesion to cartilage. Molec. Microbiol. 7, 99-107.

27 Patti, J.M., Bremell, T., Krajewska-Pietrasik, D., Abdelnour, A., Tarkowski, A., Ryden, C. and Hook, M. (1994) The Staphylococcus aureus collagen adhesin is a virulence determinant in experimental septic arrthritis. Infect. Immun. 62, 152-161.

28 Homonylo McGavin, M., Krajewska-Pietrasik, D., Ryden, C. and Hook, M. (1993) Identification of a Staphylococcus aureus extracellular matrix-binding protein with broad specificity. Infect. Immun. 61, 2479-2485. 